\title{
Grundwasser wird jünger, attraktiver und internationaler
}

\section{Nico Goldscheider ${ }^{1}$ (D) Christoph Neukum $^{2}$}

Eingegangen: 5. September 2018 / Online publiziert: 21. September 2018

(c) Springer-Verlag GmbH Deutschland, ein Teil von Springer Nature 2018

Gemeinsam mit dem Springer-Verlag, dem GrundwasserRedaktionsteam und dem FH-DGGV-Vorsitzenden haben wir uns Gedanken gemacht, wie wir Grundwasserjünger, attraktiver und internationaler machen können. Konkret haben wir drei kleine aber feine Neuerungen beschlossen:

1. Ab sofort können Manuskripte eingereicht und veröffentlicht werden, die auf herausragenden Master- oder sogar Bachelorarbeiten basieren. Diese werden durch ein neu gestaltetes Siegel des Springer-Verlags als „Exzellente Abschlussarbeit" gekennzeichnet (Abb. 1). Die Spielregeln hierfür sind sehr einfach: Der Autor der Abschlussarbeit muss Erstautor sein; Koautoren sind selbstverständlich erlaubt; die Arbeit muss ,sehr gut“ bewertet worden sein; der Abgabetermin darf zum Zeitpunkt der Einreichung maximal ein Jahr zurückliegen. Eingereichte Manuskripte durchlaufen ein reguläres Review-Verfahren und werden in eine der existierenden Kategorien eingruppiert, also Fachbeitrag, Übersichtsbeitrag oder Technische Mitteilung. Als zusätzliche Anerkennung erhält der Erstautor von Springer einen Buchgutschein, und der Artikel wird für zwei Monate öffentlich freigeschaltet (free access). Damit wollen wir zum einen den wissenschaftlichen Nachwuchs fördern, zum anderen aber auch zusätzliche, besonders spannende Beiträge für Grundwasser akquirieren.

2. Ab sofort wird es jedes Jahr zwei „Editors' Choice“-Artikel geben, d.h., die Editoren wählen zwei besonders in-

Nico Goldscheider

goldscheider@kit.edu

Christoph Neukum

christoph.neukum@bgr.de

1 Institut für Angewandte Geowissenschaften, Karlsruher Institut für Technologie, Kaiserstr. 12, 76131 Karlsruhe, Deutschland

2 B2.3 Grundwasserressourcen - Beschaffenheit und Dynamik, Bundesanstalt für Geowissenschaften und Rohstoffe, Stilleweg 2, 30655 Hannover, Deutschland

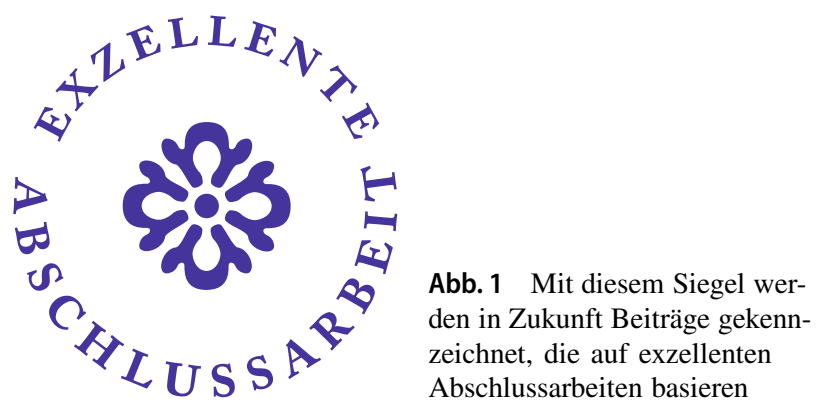

teressante, relevante und qualitativ hochwertige Artikel aus, die bekannt gegeben und vom Springer-Verlag für zunächst zwei Monate öffentlich freigeschaltet werden.

3. Neben einem englischen Abstract, einer englischen Übersetzung des Titels und den Keywords, sollen $a b$ sofort bei allen eingereichten Artikeln auch die Abbildungs- und Tabellenbeschriftungen zusätzlich auf Englisch angegeben werden. Die Richtlinien für Autoren wurden entsprechend angepasst. Damit sollen die Beiträge in Grundwasser für internationale Leser attraktiver und zugänglicher werden, denn oftmals lassen sich die wesentlichen Ergebnisse und Aussagen eines Artikels allein anhand der genannten Elemente recht gut erfassen.

Darüber hinaus weisen wir darauf hin, dass in Grundwasser wie bisher auch englischsprachige Artikel publiziert werden können. Daher sind alle unsere Leser dazu eingeladen, ihre internationalen Kollegen zu einer Veröffentlichung in Grundwasser zu motivieren, insbesondere, wenn es sich um Themen handelt, die für die deutschsprachige hydrogeologische Fachgemeinde relevant sind. 